\title{
Rolf Knieper Recht der Kondiktionen: vergebliche Versuche, Bereicherung zu rechtfertigen
}

\author{
Bereicherungen des einen auf Kosten des an- \\ deren ereignen sich ständig und allenthalben \\ Leo Weyers \\ Bereichert Euch! \\ Louis Philippe, König von Frankreich \\ $1830-1848$
}

\section{Einleirung}

Bereicherungsrecht ist eine ärgerliche Materie. Kein Autor, der sich zu ihm äußerr, versäumt, es als sverwirrend", wüberzüchtet «, wmysteriös « darzustellen', kaum eine gerichuliche Entscheidung ergeht, ohne daß sich nicht eine ausgedehnte Kontroverse an sie anschlösse. Beschränkungen auf technisch-dogmatische Informationsvermittlung versagen: selbst Repetitorien schwellen zu umfangreichen Darstellungen an und beteiligen sich an der grundsätzlichen Diskussion'. Gelegentliche Anfragen' bei Praktikern ergeben, daß jedenfalls Instanzgerichte vor der Behandlung bereicherungsrechtlicher Probleme zurüickschrecken, daß in Schriftsätzen und Urreilen nach Konstruktionen gesucht wird, die mit ihnen zusammenhängenden Komplikationen zu vermeiden, wobei nicht uninteressant ist, daß solche Konstrukcionen gelingen. Bereicherungsrecht scheint nur erwas für den BGH, OLGe, den akademischen Berrieb zu sein; emporstrebende Wissenschaltler können Fingefferrigkeir, Denken in Dreiecks-Verhältnissen mit vielfachen Abkürzungen und - mehr als heute in anderen Bereichen des Zivilrechts - Kenntnisse in der lateinischen Sprache nachklassischer Prägung belegen; für normaler Juristen kann es mit den $\$ \$ 12 \mathrm{ff} B G B$ offensichtlich überwiegend nur dann ernst werden, wenn sie einmal in eine staatliche Prüfungstommission berufen werden und dort an - überwiegend wiederum - normale Kandidaten abnorme Fragen stellen.

Der folgende Beitrag versuchr nicht, die Meinungsvielfalt um eine neue Variante zu bereichern. Die Absicht geht vielmehr dahin, die Günde der ,Verwirrung zu erklären, d. h. herauszufinden, warum sie besceht und warum sie den Fortgang der Zivilrechtspflege nicht behindert. Dabei vertrete ich die Auffassung, daß das Gewirt der Meinungen nicht auf der intellekruellen Unzulänglichkeit der BereicherungsTheoretiker beruht, sondern die Vergeblichkeit dokumentiert, die Ergebnisse der Konkurrenz von Privateigentümern nach Kriterien materieller Richrigkeit, gewissermaßen ex ante, zu bewerten.

Das heißr nichr, daß die jurisrische Diskussion irrational oder sinnlos sei; ihr Sinn

1 Vgl. nur Koppensteiner/Kramer, Ungerechtferrigze Bereicherungr 1975, 5. 20; Joerges, Bereicherungsrecht als Wirschaís reche, 1977, S. 7 .

2 Vgl. erwa Alpmann/Raddatz, Schuldrecht Besonderer Teil, Heft 3: Bereicherungsrecht, 1976.

3 Mit dieser Formulierung wird dokumentier, daß hier kein Beitrag zur inzwischen raffinienen Rechtstassachenforschung geplant isc. Mitgliedern dieser Abrsilung wird es gelingen, meine Aussagc zu varieren. 
erschließ\}t sich m. E. jedoch erst aus dem - in der zivilistischen Tradition künstlich zerrissenen - Zusammenhang des materiellen zum Verfahrensrecht: Die von Luhmann analysierte Funktion des Verfahrens, soziale Konflikre zu individualisieren, um (unrerlegene) Verfahrensbereiligte als "Querulanten« isolieren zu können', erhält durch die ernste Debatze um richtiger Lösungen des materiellen Rechts die für Legirimation unabdingbare Seriosität.

Von diesem Ansazz her läßt sich im übrigen die Verwunderung darüber auflösen, daß die bis vor einigen Jahren Furore machende , Krise der Dogmarike so sang- und klanglos von der Hochkonjunktur neuer Lehrbücher, Grundrisse, Case-books, Schwerpunkte, Einführungen, Repetitorien aufgesogen worden ist: Die Krise besteht nicht erst seit der ,Existenz von Marktmacht - wie häufig vermucet wird -, sondern sie ist von vornherein die Normalität einer Dogmatik, die materiell richriges Rechus anstrebt.

Der Absicht dieser Studie entsprechend greife ich die Bruchstellen des Kondikcionenrechis heraus:

- den Versuch, die einheitliche Grundlage des $\$ 8$ ז 2 BGB in ein Neız voneinander unabhängiger Ansprüche aufzugliedern, wobei jeweils eigenständige Werrungssysteme entworfen werden, die sich vergeblich mühen, vom rquasi-contractlichen Charakter Abstand zu nehmen - vergeblich nicht, weil im Bereicherungsrecht auch noch Grundsätze der Privatautonomie wirksam wären, sondern weil Bereicherungsrecht und Vertragsrecht unter Verkennung der ökonomischen Strukrur als Hort der Willensfreiheit angesehen worden sind (II);

- den Versuch, den Begriff des Werts ( $\$ 8$ 18 Abs. 2 BGB) hermeneuvisch zu füllen angesiches einer Waren-Zirkulation, die nur über Preise verfügt und den Wer dementsprechend nur als alcernativen Preis definieren kann (III);

- den Versuch, durch materiale Wertungen Lösungen zu legitimieren, für die der Gesetzesinhalt keine Anhaltspunkte liefert (IV).

\section{Die Sezzung von Anspruchseraussetzungen}

Die $\$ \$ 812$ ff BGB sind selbstverständlich nicht vollständig als sMysterien -Spiel zu verstehen: Wo die Folgen eines Eigentums-Uberganges trotz nichrigen obligatorischen Zrvei-Parteien-Vertrages rückgängig gemacht werden sollen, komme der Subsumierer mit der , Positivitäı des $\$ 812$ ebensogur aus wie er mir den $\$ 816 / 822$ zurechtkommt, um die Mehr-Parteien-Beziehungen im Gefolge gurgläubigen Erwerbs zu erfassen; selbst das Zusammenspiel von $\$ 935$ und $\$ 185$ BGB mache nur wenig Schwierigkeitens.

Die Komplikationen beginnen, wo die Technizität von Rückabwicklungsverhäitnissen zugunsten pragmatischer oder materialer Lösungen aufgegeben wird. Nicht nur wo das positive Recht keine klaren Anweisungen enthält, greifen die Lösungsversuche auf Wertungs-Kriterien zurück: Da der Streit um richriges Bereichenungsrecht، bereits im 19 . Jahrhundert heftig loderte, kanr die positivrechcliche Formulierung als eine Episode in der Auseinandersetzung geteen und entsprechend schnell beiseite geschoben werden: (Gesetzgebungs-) Geschichte und Wortlaut der \$8 812 If BGB gelten nicht viel: Bereits 1909 schlug Fritz Schulz in seiner bis heute einflußreichen 
Arbeit eine weire Auslegungs- und Analogien-Praxis vor, in deren Gefolge $\$ 5 \mathrm{r}_{2} \mathrm{ff}$ neu gelesen werden sollten ${ }^{6}$.

Dieser - methodologische - Betund kann als einende Klammer für die Auslegung des $\$ 812$ gelten: Unabhängig davon, ob man von einem einheirlichen Bereicherungsanspruch ausgeht und so eher dem Wortaur des $\$ 8 r_{2}$ folgr ${ }^{7}$ oder ob man aus dem dürren Wortaur ein fein ziseliertes Geäst der verschiedensten Anspruchsgrundlagen aufbauc, um für Leistungs-, Eingriffs-, Verwendungs-, Rückgriffskondiktionen verschiedene, Rationalitätenc herauszuarbeiten ${ }^{8}$ - unter keinen Umständen kommt die Diskussion um Wertungen unà Legitimations-Erklärungen herum.

Die Darstellung dieser Problematik nimmt die Unterscheidung nach Kondikuionsuntergruppen hin, obwohl die Differenzierungen mir inhalclich wenig gerechtfertigt erscheinen: Die Probleme uncerscheiden sich nicht fundamental, insbesondere sind Leistungs-, Eingriffs-, Rückgriffs- und Verwendungskondiktionen fester ans Vertragsrecht (und dies an jene) gekoppelt, als die häufigen Appelle zur Abcrennung vermuten lassen. Im folgenden wird auch deuclich werden, daß ich keinen historischen Prozeß der Abspainung des Bereicherungsrechis vom Vertragsrecht erkennen kann, sondern daß beide Gebiete ähnlichen Entwicklungen unterworfen sind, die bloßlegen, daß die überlieferte Grundannahme von der Realisierung freien Willens im Privatrecht - d. h. im Bereicherungs- wie im Vertragsreche - von vornherein nicht der ökonomischen Struktur von Wasenproduktion und -rausch entsprach, also Privatrechs auch niemals einseitig a's ,Formalrechtr existierte".

\section{a) Wertungen bei der, Leistungskondiktion,}

Für die problematischen Fälle der Bereicherung ddurch Leistung eines anderen (\$812 Abs. I Satz 1) - die Dreiecks-Konstellarionen besonders des privacen Bauund Bankrechrs - hat Canaris überzeugend nachgewiesen, daß Begriffsinterpretationen nur scheinbar auf Wertungen verzicheen. Nachdem im Laufe der lerzten Jahrzehnte über eine Reihe anderer Begriffsbildungen sich nun in der Interpretation eines smodemen Leistungsbegriffs', der als sbewußte und zweckgerichtete Vermögensvermehrung präzisiert war ${ }^{10}$, eine einigermaßen gesicherte h. L. etabliert zu haben schien, hat Canaris eben dieser Begriffsbildung vorgehalten, sie lege zunächst in den Leistungsbegriff hinein, was sie dann aus itom herauszuholen versuche'r, ein Vorwurf, dem bisher nur 'pragmatisch begegnet worden ist ". Canaris hat, nachdem jede begriffliche Interpretarion sich als untauglich erwiesen hat, den Vorschlag gemacht, die Wertungen offenzulegen, die sich hinter der Begriffsinterpretation lediglich verbergen. Er hat - neben der Zuordnung von Einwendungen - besonders die Verteilung des Konkursrisikos herausgehoben und die Auffassung vertreten, daß

\footnotetext{
6 Schulz, System der Rechte auf den Eingriffserwerb, AcP 105 (1909), S. Ifl.

7 Dafür plädieren erwa Xoppenstciner/Kramer (FN r). S. 193 fl.

8 So inzwischen dic ganz h. M., vgl. erwa Wilburg, Die Lehre von der ungerechternigten Bereicherung nach österreichischem und deutschem Reche 1934, S. I4 16 .; v. Caemmerer, Bereichcrung und uncrlaube Flandlung (1954), ziticr nach: v. Cacmmercr, Gesammche Schriften I, 1968, S. 209 lí; Larenz, Lehrbuch des Schuldrechis, Band 11, Besonderer Teil, 11. Aufl. 1977, S. 766 ff.; Esser-Weyers, Schuldrechı, Band II, Besonderer Teil, Teilband 2, 5. Aufl. 1979. S. 29 ff.; Joerges (FN 1) S. 62.

9 Insolem besteht eine Differenz zur Einschäızung der Vcränderung des Zivilrechrs, wie sie Voegelj lürs Deliktsrecht in dieseon Helt vomimmt.

10 Vgl. etwa Koppensteiner/Kramer (FN t) S. 2s; Larenz (FN 8) 5.467.

1) Canaris, Der Bercicherungsausgleich im Dreipersonenverhälınis, Festschrifl für Larenz, 197\}, 5. 799 (f.

12 Esser-Weyers (FN 8) S. $38 \mathrm{Ht}$.
} 
dieses in die Sphäre der jeweiligen frei und eigenverantwortlich handelnden Vertragspartner zu plazieren sei. Aus dieser wertenden Abwägung ergebe sich eine angemessene Lösung der Dreiecks-Konstellationen'3. Der Beitrag von Canaris bezeichnet m. E. die Grenze, bis zu der juristische Dogmarik vorzustoßen vermag. Gleichzeitig bezeichnet sie die Begrenztheit eines zur Begründung von gerechten Lösungen auf Wertungsgesichrspunkte zurückgreifenden Obligationsrechts. Canaris muß am Axiom privatautonomer Eigenverantwortlichkeit festhalten, obwohl die wechselseitige ökonomische Abhängigkeit in den Dreieckskonstellationen gut zum Ausdruck kommt: In einem Konkurs manifestiert sich nicht individuelles Fehlverhalten individueller Vertragspartner, sondern der Zusammenbruch vielfältiger $\mathrm{Be}$ ziebungen von Lieferanten, Abnehmern, Kreditgebern und -nehmern in einer durch konjunkturelle und strukturelle Unsicherheit gekennzeichneten Okonomie. In einem 'Baukonkurs''4 sind Bauherr, Baustofflieferant, Bauträger, Bauunternehmer, aber auch der Staat und Kredicinstiture insgesamt verwickelt, und niches anderes gilt für die Bankanweisungsfälle's. Die konkrete - und häufig ganz zufällige - Vertragsbeziehung zur Anknüpfung der Risikozuschreibung zu machen, vernachlässigr diese enge Verknüpfung und konstruierr künstlich individuelle (Eigen-)Verantwortlichkeiren, wo soziale Abhängigkeiten vorliegen, die im übrigen weir über die Dreiecks-Beziehungen hinausragen. Die theorecische Aussage von der Unzulänglichkeit der Konkurstheorien als , Bestrafung c für individuelles betriebswirtschaftliches Fehlverhalten ${ }^{16}$ ist inzwischen empirisch gur belegr ${ }^{17}$, so daß Wertungen, die auf diesen Theorien aufbauend individuelle (Bereicherungs-)Risiken verteilen, nichr nur im Ergebnis nicht viel weiter reichen als Versuche, über den Leistungsbegriff Zweier-Beziehungen aus Vielfach-Beziehungen zu isolieren.

\section{b) Wertungen bei der Bereicherung in sonstiger Weises}

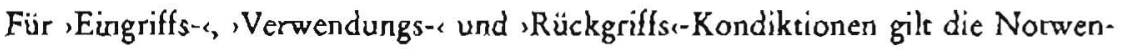
digkeir von Wertungen ebenfalls, wegen der unpräzisen Fassung des $\$ 812$ und des weiten Anwendungsfeldes für diesen Bereich in noch stärkerem Maße. Obwohl sters versichert wird, es handele sich bei den $\$ \$ 1 \mathrm{r}$ ff nicht um ein $n$ Recht höherer Ordnung * oder um allgemeines Biligkeitsrecht ${ }^{172}$, greifen Rechtsprechung und Lehre doch immer wieder auf Billigkeitserwägungen, Treu und Glauben und ähnliche offene Wertungen zurück. Das Scichwort bat bereits der, GleisanlagencFall des Reichsgerichts' ${ }^{8}$ geliefert, wo unter "Billigkeirserwägungen" ein Bereicherungsanspruch zugesprochen worden war, wenn der Bereicherre bei wordnungsgemäßem Vorgehen « sich auf einen entgeltlichen Vertrag hätte einlassen müssen.

Im Taxi-Parkplarz-Fall'19 akzentuier sich diese Auffassung: Der Eigenrümer eines Bahnhofsvorplarzes harte von den dort regelmäßig parkenden Taxiunternehmern eine Vergütung haben wollen und ein entsprechendes Vertragsangebor gemacht. Nachdem die Taxiunternehmer das Angebot abgelehnt hatten, klagte der Eigentümer die im Vertragsangebor vorgeschlagene Summe als Anspruch aus $₫ 81 z$ ein. Der

1) Canaris (FN I1) S. 802 If 1850 ff.

14 Vgl. erwa die Fallkonsedlation in BGHZ $\{6,228$.

is $V_{\mathrm{gl}} \mathrm{erwa}$ die Fallkonstellation in $\mathrm{BGHZ} 67,78$.

16 Austübrlich Knieper, Konkurs und Sanierung, BB 1977, 622.

17 Gessner u. 3., Die Praxis der Konkursabwicklung in der Bundesrepublik Deutschland, 1978, bes. S. $522 \mathrm{ff}$.

$172 \mathrm{Vgl}$. etwa v. Caemmerer (FN 8) S. 115.

18 RGZ $97,310$.

$19 \mathrm{BGHZ} 20,270$ 
BGH erkannte die Summe in voller Höhe an, da "bei ordnungsgemäßem Vorgehen

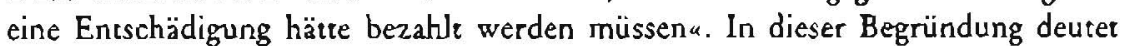
sich an, daß der Billigkeitsgesichtspunkt aus der Weigerung, einen Vertrag abzuschließen, abgeleitet wird. Vom Ergebnis her fehlt zur anderen »Parkplasz«-Entscheidung des $\mathrm{BGH}^{20}$, wo trorz ausdrücklich gegenteiliger Erklärung eines Parkplatzbenutzers gegenüber dem Vertreter des Parkplatzeigencümers aus ssozialtypischem Verhalten, ein Vertrag abgeleitec worden war, jeder Unterschied. Aber auch die Begnündung operiert in beiden Fällen mir Vertragselementen, so daß die Hoffnung, die Lehre vorn faktischen Vertrag durch eine stärkere Betonung des Bereicherungsrechts überflüssig machen zu können, nicht abwegig ist, wohl aber die Hoffnung, mitrels dieses Konstrukrionstricks die Reinheit der Privatautonomie zu erhalten ${ }^{2 x}$.

Steckt man das Argumentationsfeld genauer ab, so ergibe sich, daß die Differenzen zwischen (faktischem) Vertrag, Leistungskondiktion und Eingriffskondiktion von ibren theoretischen Begründungen her außerordentlich gering sind, so daß das Beharren auf strikter Differenzierung der verschiedenen Kondikrionsarten auf den ersten Blick zweifelhaft scheint. Darauf wird zurückzukommen sein. Woher aber nehmen die Encscheidungen ihre Kriterien für Billigkeit? Warum ist es »mir Recht und Billigkeit nicht zu vereinbaren «, wenn jemand ein Produkc herscellt, das auch ein Konkurrent herstellt, dem ein Gebrauchsmusterrecht eingeräumt worden war, um vor Konkurrenz während der Schurzperiode gesicher zu sein" Modeneuheiten imitier ${ }^{23}$, wenn ein Taxi sich unentgeltlich auf dem Bahnhofsvorplatz aufhält, wenn eine Gleisanlage ohne Entgelterhöhung intensiver als ursprünglich angenommen genutzt wird? Sicherlich handelt es sich jeweils um Konstellacionen, in denen der Bereicherte, hätte er sich auf Vertragsverhandlungen eingelassen, ohne vertragliche Entgeltsverpflichtung nicht davongekommen wäre. Aber ein solcher Vertragsschluß liegı nun gerade nicht vor, in einigen Fällen weigerte sich der Bereicherte sogar ausdrücklich, ein Entgelt zu zahlen. Die Begründung der Billigkeit mit dem Argument, ein ordnungsgemäß Handelnder hätte sich auf einen Vertragsschluß einlassen müssen, widerspricht deshalb ebenso dem Grundsatz des Respekrs des sfreien Willens, und der Privatautonomie, der in der herrschenden Rechtsprechung und Lehre neben dem Bereicherungsanspruch verreidigt wird, wie die Rechtsprechung zum faktischen Vertrag, die sich aber praktisch trotz solchen Widerspruchs hält.

Nun läßt sich nicht leugnen, daß es mir in meinen menschlichen Momenten auch anständig vorkomme, daß der Modeneuheitenimitator, der den Bahnhofsvorplatz zur Geschäfusanbahnung ausnuczende Taxifahrer, der ein fremdes Gebrauchsmuster zum eigenen Vorreil Nurzende zur Kasse gebeten werden. Wenn ich aber diesem Instinkt nachgebe, wenn ich meine privaten Anstandsvorstellungen auf juristische Fallösung überfließen lasse, dann beginne ich Bereicherungsansprüche zu konstruieren, wo sie niemand für gegeben hält: Dann meine ich, es sei anständig, wenn ein Gastwirt, der vom Zustrom never Gäste proficiert, weil der Blitz den einzigen Konkurrenzgasthof am On getroffen hat, seinen ebemaligen und jetzt verarmten Konkurrenten am Gewinn beteiligen solite, desgleichen ein Unternehmer, der mit einer großangelegten Werbekampagne s0\% der Kunden seines Konkurrenten zu

20 BGHZ $21,319$.

21 In diesem Sinne aber Mestmäcker, Eingriffserwarb und Recbusverleizung in der ungerechrenigzen Bercichcrung (1958), zitiert nach: Mestmäcker, Recht und ökonomisches Geselz, 1978, S. 451 ff.

12 BGHZ $88,90 / 99$

2) Tatsïchlich häle der BGH einen Anspruch gegen den Imicator von Modeneuheiten. für gegeben: BGHZ 60, 168 . 
sich herüberzieht, desgleichen ein Verkäufer, der mit überlegenem Verhandlungsgeschick einen besonders günscigen Preis für sich heraushandelt ${ }^{24}$. An diesen Beispielen wird deutlich, daß Anständigkeitsgefühle vom rechten Wege abbringen: Verhandlungs-, Werbungs- und Durchsetzungsgeschick, eingesetzr, um eigenes Vermögen zu mehren, gehört ja gerade zu den Tugenden des Geschäftsmannes, wie sollten darauf aufgebaute Bereicherungen auf den Sand der $\$ \$ 8$ z $\mathrm{ff}$. gebaut sein: "Bereicherungen des einen auf Kosten des anderen ereignen sich scändig und allenthalben $a^{2 s}$ - in der Tat: Dieser Satz ist nur die rechtstatsachenmäßig formulierte Variante des Schlachtruls der nach-revolucionären franzsösischen Restauration, des »Bereichert Euch!«, der über đas revolucionäre 'Freiheit, Gleichheit, Brüderlichkeite den Sieg davongetragen hat.

Ais Nebenbemerkung gegenüber einem großen Wort sei der Hinweis gestattet, daß der Schlachtruf nicht hieß: "Bereichert Euch im Rahmen der Kondikrionsregeln! $*$. Daß es sich dabei niche nur um eine Auslassung aus Elfekıgründen handelt, wird bereits an der oberflächlichen Beobachtung deudlich, daß Menschen in unserer Gesellschaft hohes Ansehen genießen, die (oder deren - euphemistisch - Vorfahren) ibr Vermögen zusammengegaunert, - gemordet, -geraubt oder -geheiratet haben, also keineswegs als ,Leistende، im .Leistungswertbewerb. Im volkstümlichen Respekt, der solchen Menschen entgegengebracht wird und der sic nicht selıen in hohe Regierungsämter trägt, dokumentien sich wohl nicht nur Dummheit, sondern die wcise Einsicht, daß es mit derartigem Vermögen nur abgestuft anders bestellt ist als mit dem im - Lcistungswetibewerb, Erworbenem: Bereicherung der einen, wenigen auf Kosten der andoren, vielen findes allemal stats.

V. Caemmerer har diesen Tatbestand an einem Beispiel aus der Welı des Handwerks erläutert, es sei *klar «, daß die Leistung eines abhängig beschäfrigten Glaserlehrlings weine Leistung des Glasers ist, der über die Arbeitsleistung, die er von seinem Lebrling zu fordern hat, natürlich disponieren kann $\alpha^{25}-$ Naturrecht!

Nachdem auf diese Weise eine eherne Regel für wdie Verteilung neu produzierter Werte ${ }^{2 y}$ besteht, findet die Frage nach dem Ausgleich von Bereicherungen quasi eine Etage höher statt, und obwohl Zweifel an der Tauglichkeit der Ansrändigkeit bei der Lösung bürgerlich-rechtlicher Streitigkeiten geäußert worden sind, beharr der BGH darauf, daß es in dieser Erage Anstand und Sitce gibr. Nehmen wir, um die Tragfähigkeit dieser Auffassung zu prüfen, den unlängst entschiedenen „Gebrauchsmuster $\alpha-F a l l$ auf ${ }^{28}$. Der Fall ist bereits deswegen interessant, weil mit ihm eine Rechtsprechungs-Umkebr vollzogen wurde, eine Art Kapitulation des BGH vor einer überwältigenden - in Steigerung zu herrschenden - Literacurmeinung. Die Entscheidung nimm denn auch eher Zuflucht zum ausführlichen Zitat dieser Literatur als eine eigene Begründung vorzutragen. Diese fäll lapidar mit dem Satz aus, daß es eben mmit Recht und Billigkeit nicht zu vereinbaren (sei), daß der Schurzrechtsverleszer unangefochten behalten soll, was er durch eine widerrechrliche (nicht schuldhafte - R. K.) Verletzungshandlung erlange hat *.

Damit ist nichr nur eine Billigkeits- und Anstandsregel positiv definiert, sondern zugleich gesagt, daß die RG- und BGH-Richter, welche die Gegenmeinung vertreten, von Billigkeit und Anstand keine korrekte Vorstellung hatten. Ehe wir ein solches Verdikt unterstützen, wollen wir annehmen, daß die Kriterien für Billigkeit und Anstand nicht einfach zu entwickeln sind. Die jeczt überholte Auffassung harte sich im wesenclichen auf zwei Ǔberlegungen gestützr. Zum einen schloß sie aus der Existenz der gegenüber $\$ 823$ BGB sondergesetzlichen Schadensersatzregelung in

24 Diesen Fall diskucicr schon v. Savigny, System des heurigen Römischen Reches, 5. Band, 1841 ، S. 526.

25 Esser-Weyers (FN 8) S. 29.

26 v. Caemmerer (FN 8) S. 227 l.

27 joerges ( $\left.\mathrm{FN}_{1}\right) \mathrm{S}_{2} 23$.

28 BGYZ 68, 90. 
\is GebrMG, die Verschulden als Anspruchsvoraussetzung enthält, daß Ansprüche aus dem BGB, also auch aus Bereicherungsrecht, im Wege der Spezialkonkurrenz ausgeschlossen sein sollcen. Zum anderen gewähre der Staat mit Gebrauchsmustern begrenzte Rechte und konstruiere Gürer, die - wie es Kohler für Immaterialgüterrechte und insgesamt ausgedrückc hacte - - doch der Allgemeinheit zuscreben * und deren Ausgleichung die nLebensumstände unnötig verbittern « müßren ${ }^{29}$. Endlich wurde sbefürchter, der Patencinhaber (wo die Rechtslage idencisch ist $-\mathrm{R}$. K.) könne mir dem Bereicherungsanspruch Mißbrauch treiben, indem er ruhig zusehe, wie ein anderer aus seiner Erfindung Gewinn ziehe, den er alsdann beanspruche so. $^{\circ}$.

Wieder verfängt offensichtlich das Argument von Wortlaut und Geseczessystemacik wenig, obwohl es im Patentrecht durch die Gesetzgebungsgeschichte unterstützt wird: Die Reichscagskommission für das Patentgeseız hatce, wie Schulz in seinem Plädoyer für die Anwendung des $\$ 812$ erwähnt", sich ausdrücklich gegen die Zulassung von Bereicherungsansprüchen ausgesprochen. Entgegen allen Subsumrionsrezepten findet die Auseinandersetzung wesentlich auf der Ebene von Wertungen start. Schulz, der als eigentlicher Begründer dex Lehre vom Bereichenungsanspruch bei Immacerialgüterrechten gelten kann, fand es sunerhört "s2 und dem "Rechtsgefühl ${ }^{3}$ " widersprechend, den Anspruch zu versagen. Er hat vorgeschlagen, $\$ 812$ de lege laca neu zu schreiben und an jeden $\times$ Eingriff in ein subjekcives Privatrecht...., das der Betrolfene sich nicht gefallen zu lassen braucht $"$, an rechtswidriges Handeln also, Bereicherungsansprüche zu knüpfen ${ }^{34}$. Diese Aulfassung geht weit. Sie schützt nicht nur die Monopolstellung des Patents, des Gebrauchsmusters, des Geschmacksmusters, des urheberrechtich geschützien Werkes, des Warenzeichens, sondern auch den Träger des Persönlichkeitsrechts sowie des - Rechts auf ungestörten Gewerbebetrieb, ein Recht, durch Betrieb des Gewerbes ein Einkommen zu erwerben $«$. Dieses von Schulz frei definierce Recht, das durch das UWG geschützc sei, gehört sin die Nähe der Aneignungsrechre "s". Damit sollen Vermögenspositionen individuell zugerechnet und zementiert und scarische Besitzstände aus der Konkurrenz herausgehalten werden, wie es sich kaum noch mit der „Kampfordnung des Wettbewerbs ${ }^{36}$, die von der Enteignung der Eigentümer durch gerissenere und mächrigere Eigentümer, von der Verfolgung des 'privaten Lasters zur Herstellung des öffentlichen Wohls، (Mandeville) lebt, vereinbaren läßt. Dementsprechend ist - wesentlich durch Wilburg ${ }^{37}$ und v. Caemmerer ${ }^{18}-$ mit der Lehre von der Güterzuordnung versucht worden, an die Stelle einer Handlungsbewertung die Bewertung von Vermögenszuständen zu setzen: „Das Interesse des Eingreifers an freier gewerblicher Berärigung ist von prinzipiell gleichem Rang wie das des Rechrsinhabers «' 39 . Es ist gebilligt und gerechtfertigt, "Marktpositionen und Gewinne auf Koscen anderer Markteeilnehmer zu erlangen ${ }^{\star 2}$. Nur wo sabsolute

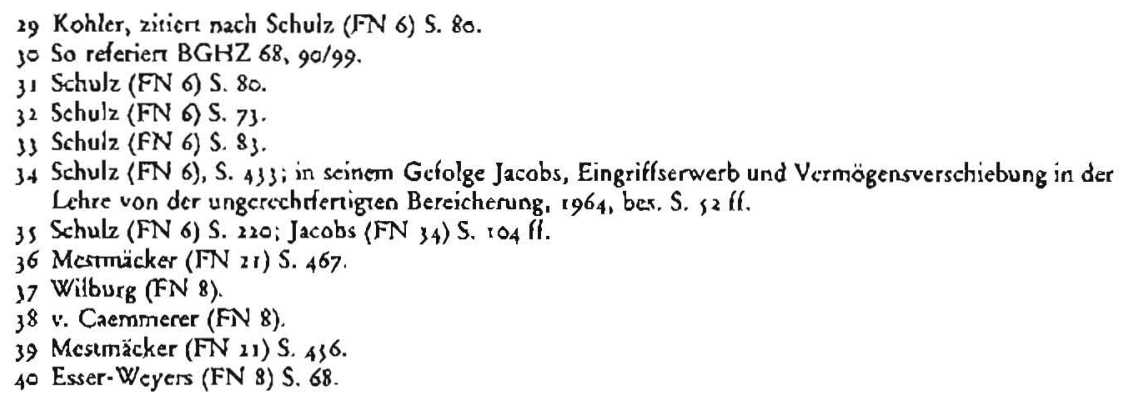


Rechte und absolut geschützze Interessen «" verletzt sind, sollen Ansprüche aus $\$ 8 \times 2$ gegeben sein. Der wrein sachliche $Z_{w e c k}$ des verkürzten Rechtes, bescimmte Gürer und deren Nurzen dem Berechtigren zuzuweisen, scheint das Geheimnis der Ungerechterrigkeit fremden Erwerbes zu sein $\alpha^{42}$. Trotz der ausgedehnten Debatte ist dieses Geheimnis nicht gelüfret, und obwohl bereits Wilburg den Hinweis auf "Billigkeit und Gerechtigkeit « angesichts seiner Verfeinerungen des Wissensstandes als «ausgediente Helfer « abtun wollte ${ }^{43}$, kehrt der BGH im "Gebrauchsmuster *-Fall trotz seiner generellen Zusrimmung zur Zuordnungsgehaltslehre genau dahin zurück, wenn er es ebenso empört wie Schulz für unmöglich hält, daß jemand etwas recheswidrig Erworbenes behalten können soll.

Diese Entscheidung im Einzelfall triffr sich nun trorz der unterschiedlichen theoretischen Ausgangsposition mit den Verfechtern der Lehre vom „Zuordnungsgehalt«. Obwohl für Konkurrenz, insbesondere in der Form der Leistungskonkurrenz, Raum offen gehalien werden soll, stehr diese Lehre seir Wilburg auf dem Standpunkt, Schulz habe jedenfalls Recht, wenn er bei vernragsloser Nutzung fremder Immaterialgüterrechce Bereicherungsansprüche eirräume ${ }^{44}$. Auch die Akzentuierung dieser Lehre im Sinne des Ordo-Liberalismus beklagt dieses Ergebnis nicht. Nachdem der Kampf gegen die Einräumung von Monopolstellungen durch Immaterialgüterrechte einmal aufgegeben ist, ja nachdem die sonst als leistungshemmend angegriffene Monopolstellung auf wunderbare Weise als "Ermuntenng der Unternetumen zur Verbesserung der Leistungen « qualifiziert isc"s, fällt der Anschluß an die Lehre von der extensivsten Interpretation und Nutzbarkeit dieser Monopolstellungen leicht; die Warnung vor der sertragsfalle hat bei Mesumäcker keine prinzipielle Bedeutung mehr, sie wird nicht als Grund zur Ablehnung von Ansprüchen ausgesprochen, sondern wesentlich nur noch als Kriterium zur Unterscheidung von vertraglichen und Bereicherungsansprüchen ${ }^{46}$. Lediglich in Randbereichen wird das Interesse an freier gewerblicher Berätigung، (Mestmäcker) geschürzt: so etwa bei der Nutzung von fremden Warenzeichen, beim Eingrilf in Persönlichkeitsrechte oder den eingerichteten Gerverbebetrieb47.

\section{c) Wertungen beim konstruierten Wegfall der Entreicherung}

Wer einen Bereicherungsanspruch bei der Inanspruchnahme von gewerblichen Schurzrechten oder von Dienstleistungen zusprechen will, steht vor der Schwierigkeit, daß eine Vermögensübertragung häufig nicht vorliegt: Der Vermögensmehrung stehen Vermögensminderungen nichr gegenüber. Seit der Arbeit von Schulz, die bedenkenlos vom Ergebnis diktiert war, den gewerblichen Rechtsschurz vollständig ins Zivilrecht zu integrieren, ist die Tarsache häufig fehlender Entreicherung mir der snormativen Kraft des Fakrischen a als Auslegungsmodalität - wenn auch gegen anhaltenden Widerstand $-{ }^{48}$ dogmatisch fixiert: Inzwischen genügr die Mera-

41 Wilburg (FN 8) S. 46; ähnlich v. Caemmerer (FN 8) S. 228 If; vgl, auch Burgbacher, Warenzeichenlizenzverräge im Rechr der Weubewerbsbeschränkungen, 1971, S. 61 II

42 Wilburg (FN 8) S. 27.

43 Wilburg (FN 8) S. 181 .

44 Wilburg (FN 8) S. 40 fí; v. Caemmercr (FN 8), S. 230 if.

4) Steindorf, Eindührung in das Wirtschaftsrecht der Bundesrepublik Deutschiand, 1977, S. 88; cbenso Mestmäcker (FN 21 ) S. ${ }_{4} 60$.

46 Mestmäcker (FN 2I) S. 459 lf.

47 Mestmäcker (FN 2r) S. 463

${ }_{4} 8 \mathrm{Vgl}$. z. B. J. Willhelm, Das Merkmal waul Kosten " als notwendiges Kriterium der Leistungskondition, JuS 1973, Iff. 
pher, daß es um "Bereichungsrecht, nicht Entreicherungsrecht $\alpha^{19}$ gehe, um die Schwierigkeit zu überwinden, daß der Wortlaut des $\$ 812$ mic dem Merkmal saut Kosten، durchaus auf die Vermögenslage des Berechtigten abstellt. Wo argumencier wird, spielt die Geserzesgeschichte eine ungewöhnlich wichrige Rolle: Daß es nicht - wie ursprünglich vorgesehen - saus dem Vermögen،, sondern - schwächer - sauf Kosten، heißt, gilt als Beleg zugunsten des Esserschen Merkspruchs und als Grund, auch das rauf Kosrene mit für überflüssig zu erklären ${ }^{\circ}$.

Diese Auslegung contra legem schützt den Eigentümer, den In-Haber, den durch das Monopol der gewerblichen Schurzrechre Begünstigten. Sie ist damir durch ein bei Juristen verbreitetes "Rechrsgefühl "s' geleitet, die Statik des Eigentumsschurzes, des ,wohlerworbenen Rechtes، gegen die strukturelle Unsicherheit der Marktökonomie zu verteidigen. Wie frag würdig dieses $>$ Rechtsgefühl und der in ihm aufgehobene Interessenschutz ist, zeigt sich daran, daß auf dex Grundlage von Keynes' theoretischen Uberlegungens" leicht ein alternatives ,Rechtsgefühl aufgebaut werden könnte. Wenn Veranlassung und Neigung zur Investition zu den ebenso zentralen wie nicht-selbstverständlichen Akcivitäten in einer durch Unsicherheit gekennzeichneten Gesellschaft gehören's, dann ist es angemessen, auch juriscische Gefühle für den ,Aktivens, den Investierenden, den Handelnden zu entwickeln und sie vom Eigentümer abzuziehen. Dieses, Gefühl härte allen Anlaß, auf der Entreicherung zu beharren (und sich so im Einklang mic der Geseczesformulierung zu verhalten), d. h. die Frage zu prüfen, ob der Rechesinhaber selbst gehandelt und einen Vermögensvorteil erzielt hätte, wenn ihm der Anspruchsgegner nicht zuvorgekornmen wäre. Der Rechtsgefühlssatz, daß der Verletzte nicht zu belegen brauche, er selbst habe sein Recht gewinnbringend nuzzen können, erweist sich in dieser Sicht als nicht nur durch den Wortlaut niche gedeckt, sondern auch als wirtschaftspolicisch irregeleiter.

Entsprechend und wiederum im Einklang mit dem positiven Recht erschiene es im Anschluß an Keynes angemessen, die Beschränkungen der Ersazzansprüche bei der Inanspruchnahme fremder gewerblicher Schutzrechte ernst zu nehmen und restrikriv zu interpretieren. Wie Schulz es für verschwenderisch gehalten hatte, die unter Verletzung von Patentschutz hergestellten Gegensiände zu vernichten (wie es 1909 noch in einigen Gesetzgebungen vorgesehen war) und scatcdessen vorschlug, einen Gewinnherausgabeanspruch an die Stelle zu setzen ${ }^{\text {4 }}$, so verschwenderisch muß es den Keynesianern erscheinen, dem Inhaber einen Gewinn in Form einer Art Monopolrente zuzuschanzen, bloß weil er Inhaber ist und obwohl er nicht inve-

49 Esser-Weyers (FN 8), S. 74.

so Vgl. erwa Beuthien in Srudienkommentar zum BGB, 2. Auf. 1979, $\$ 812$ Anm. $1_{4}$ : Schulz (FN 6) (z. B. 5. 479) hanc noch offener von einer neven Lesart des $\$ 812$ gesprochen.

$s_{1}$ So in dicsem Zusammenhang deutlich Schula (FN 6) S. $8_{3}$.

\$2 Wesentlich niedergelegr in J. M. Keynes, Allgemeinc Thcorie der Beschäftigung, des Zinses und des Geldes; das Werk stammi von 1936, muß also nicht erst noch für juristen bekanntgemacht werden. Keynes war nun weder der Meinung, daß sich seine Vorstellungen in der Konkurrenz der Privatcigenrumer van Geld und Kapical, d. h. ohne staatlichen Befehl, durchseczen lasse, noch daß damir Krivericn für eiren richtigen gesellschuftlichen Zusammenhang vorlägen. Bescheiden lormuliene er, daß er die Luxusausgabe lür immerhin weniger schädlich hale als die Rüstungsausgabe (S. 1 10). Er hatte seinen akruellen politischen Beitrag an die Aulrüstungsmilitärs, -polizikcr, -industriellen des Jahres 1936 gericbect. Das Jahr 1980 bictet allen AnlaB, in resigniertem Entserzen die Hollnungen Keynes' erneut als naiv zu kennzeichnen: Nachdem in den letzecn Jahren Geld in das unproduktive und investicions. bemmende Halten von Gold und anderen sSachwerzen gesteckt worden ist, scheint sich im Januar 1980 der Trend umzukehren: die selbsihcilenden Kräfte des Markies haben geschaffi, daß aus dem Gold wieder in invescive Anlagen umgesuegen wird bevorzugter Anlagewern sind die Papiere der Rüsrungs. industriv: Das alsc Rezep! kapitaliscischer Vollbeschafügungspolicik und anschließender Massenvernichrung bleibr in Ebren.

s) Keyncs (FN 52) S. $\mathrm{rr}_{4} \mathrm{H}$

34 Schulz (FN 6) S. 49 ff. 
stiert hat. Dementsprechend wäre es im Anschluß an die ältere Lehre und Rechtsprechung sbillig،, bei schuldlosen Patent-, Gebrauchsmuster- und sonstigen Immaterialgüterrechtsverletzungen einen Bereicherungsanspruch abzulehnen. Die OrdoLiberalen, denen an sich schon die Gewährung von solchen Schutzrechten suspekt sein sollre, könncen sich hier ausnahmsweise einmal mit den Keynesianem zusammentun und nicht - im Zurückschrecken yor einer massiven juristischen Lehrmeinung - sich mic Randkorreknuren ecwa des Warenzeichenrechis begnügen, sondern das investitionsfeindliche Berejcherungsanspruchs-Necz im gewerblichen Rechrsschutz insgesamt zerreißen,

Auf dieser Linie läge es endlich, den Inhalt des $\$ 818$ Abs. 3 ernst zu nehmen und ,Luxus -Bereicherungen vollsündig von der Ausgleichspflicht auszunehmen, d. h. nicht der etwa im Flugreise-Fall vom BGH vorgezeichneten Richtung einer restriktiven Incerpretarion zu folgen.

\section{Die Anspruchsböbe als Preis-Frage}

Die Diskussion um die Anspruchsvoraussetzungen hat die Vergeblichkeit dokumenriert, die in der Konkurrenz zufließenden Vermögensvorteile nach Krizerien materialer Richtigkeit individuell zuzuordnen. An diesem Ergebnis ändern auch die wirtschaftspolitischen und interessengeleiteten Fundierungen von Rechrsgefühlen nichts: Die Vermögensverteilung verschließr sich einer Charakcerisierung nach ,Richtigkeit.

Dieses strukcurelle Dilemma setzc sich fort, wenn es um den Umfang des Herauszugebenden gehr, sobald dieses nicht in der Rückerstattung eines körperlichen Gegenstandes besteht. Bei der Interpretation des $\$ 8 \mathrm{r} 6$ erhält die Glätte des Dogmatik-Bestandes ihre erste Fraktur, die sich bei $\$ \$ \$ 18$ Abs. $2 / 812$ zum tiefen Bruch enweiter. Während für $\$ 816$ die Meinung, der erzielte Preis sei herauszugeben, noch mit dem Hinweis auf die Formulierung - das 'Erlangter - herrschen kann und so die Faksizität von Preisen als einzigem Indikator für Nützlichkeit hinnimmt, zwingt die Formulierung des $\$ 818$ zur Auseinanderseczung mic dem Begriff, Werk. Der Dreiklang sobjektiv - subjektiv - vermitrelnd, ais Interpretationsmuster muß sich hier schaudernd zurückziehen angesichts einer Gesellschaft, die schon das Nachdenken über die Entstehung und Produktion objekriver Werte mit dem Geruch des Umstürlerischen belegt. Das Schaudern zeigt Wirkung. Dankbar ist - bei allen sonstigen Unstimmigkeiten - die bündige Aussage von Schulz angenommen worden, es sei wunmöglich, den Ertrag auf die Ertragsfaktoren ursächlich zurückzuführen", sowie seine Formulierung der Übernahme der Grenznurzentheorie, die jede "Zurechnungsidee . . ökonomisch unhalrbar finder, "weil sie die Wertschärzung der Konsumenten als eine Ursache des wirtschafllichen Ertrages zu wenig beachteta". Nachdem auf diese Weise die Frage nach der Wertschöpfung, die im 9. Jahrbundert unter dem Eindruck der klassischen Nationalökonomie die Diskussion bestimm bacte und noch in die Formulierung des $\$ 818$ hineinragr, auf die Ebene des Marktes und damit dogmatisch-juristischer Handhabbarkeit transponiert ist, konnte der Streit darum entbrennen, ob der *Verkehrswert « oder der individuell erzielte Kaufpreis die Interpretation des Weres bestimmen soll, wobei der Verkehrswert nichts weiter als ein effekziver oder fiktiver Durchschnittspreis ist.

Nachdern das Problem der Wertbescimmung auf den Vergleich verschieden zu 
errechnender Preise reduziert ist, verflacht die Diskussion zur Abwägung von Individualpreis, Durchschnittspreis und Aufteilung der Differenz von Durchschnitus- und Individualpreis. Der Streit geht nur noch darum, ob bei der Bewertung von ohne rechtlichen Grund erlangten Dienstleistungen, bei Wertersatz nach VeräuBerung eines ohpe rechtlichen Grund erlangten Gegenstandes der besonders geschickt herausgehandelte (Kauf-)preis, der Durchschnitspreis herauszugeben sei oder ob eine Aufteilung vorgenommen werden müssest. Das starke Wor eines Konflikrs zwischen "Arbeit und Kapital ${ }^{37}$ verdünnt sich in dieser Auseinandersetzung zum Konflike zwischen Kaufmann (= Arbeic) und Eigentümer (= Kapital). -Interessen--jurisprudenz heißt auf diesem Stand nicht mehr, als daß der Anwalt des Kaufmannes zugunsten der Interessen des Kaufmannes, der Anwalt des Eigentümers zugunsten der Interessen des Eigentümers zu argumentieren hat, und der Richcer in óer mißlichen Lage des Entscheidungswwanges sich den Interessen einer Parei zuwenden muß; wen wunderts, daß es da manchmal den einen, manchmal den anderen triffc.

Die Abwägungsnotwendigkeit zwischen individuell vereinbartem und durchschnittichem Preis taucht nicht auf, wo eine Preisabrede fehlt. Während sich der lang tobende Streit bei Vorhandensein von Abreden im Moment eber zugunscen derer entscheidet, die die akcuelle Abrede als Unter- und Obergrenze der Wertbestimmung ansetzen wollen, orientiert sich die Rechtsprechung bei fehlender Abrede mic dem Begriff wangemessene Entschädigung “ ${ }^{33}$ an Durchschnittlichkeit und Verkehrswert, wobei individual-vertragliche Elemente eine Rolle spielen können: Im Taxi-Parkplarz-Fallso ist als angemessene Entschädigung gekennzeichnet worden, was der Kläger in Vertragsverhandlungen angeboten hatte.

Wer sich erst einmal auf die Ebene des Vergleichs von Preisen auf verschiedener Berechnungsgrundlage begeben hat, für den klinge der aquasi contractlicher (v. Savigny) Gedanke einleuchtend, daß bei Leistungen, die einen Verkehrswert haben, ... grundsärzlich davon ausgegangen werden (kann), daß dieser Preis auch vereinbart worden wäre ${ }^{60}$. Bereicherungsrecht präsenciert sich insoweit wiederum trotz aller gegenteiligen Beteuerungen ${ }^{61}$ als Fortserzung des Vertragstechts mit anderen Mirteln. Der BGH folgert, daß der Wert einer Flugreise "die für den Flug üblicherweise zu zahlende Vergürung " sei ${ }^{6_{2}}$. Auf dieser Basis wurde ein beschränkr geschäftsfähiger Jugendlicher, der sich durch die nachlässigen Kontrollen der Lufrhansa gemogelt und seinen Udo-Lindenberg-Traum vom Abhauen nach Amerika wahrmachen wollte, verpflichter, der klagenden Fluggesellschaft den vollen Linienflug-Preis zu zahlen (obwohl die Maschine nicht ausgebucht war, der Schüler die Reise ohne Mogeln nie hätre antreten können und er sofort von den US-Einwandungsbehörden zurückgeschicks wurde). Canaris, der die Entscheidung im Ergebnis ablehnt, hat dem BGH bei der *Werc-Bescimmung sekundiert: Auch er definiert den Wert als "normalerweise« zu zahlenden Preis, als "Marktpreis « eben ${ }^{6}$.

Damit dokumentieren Rechrsprechung und Lehre, daß ihnen im zähen Ringen um die Gleichsetzung von Wert mit irgendeinem Preis entgangen ist, daß es eine

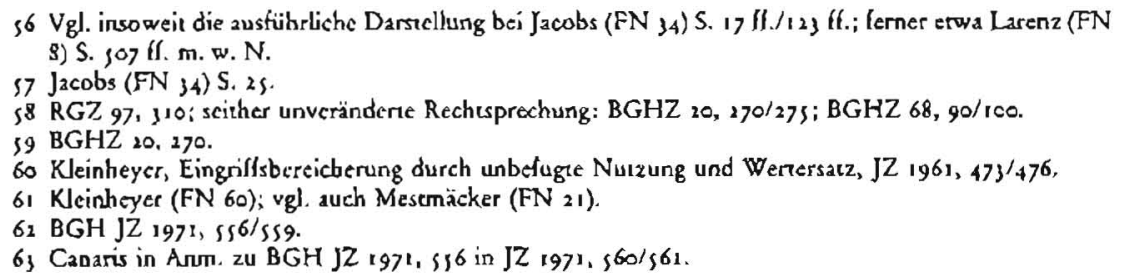


anhałrende inter- wie intradisziplinäre Diskussion um die Möglichkeiren der -Marktpreisu-Festlegung bei fehlendem Markt gibr. Die Tatsache, daß es im Flugreisegeschäft einen >Markt kaum gibt, daß die klagende Fluggesellschaft einem weltweiten Kartell (IATA) angehört, das die Flugtarife welrweit administriert, hätte selbst den subjekrivistischen Preistheoretiker zögern lassen, das üblicherweise Verlangte für das Angemessene zu halten. Auch juristische Teildisziplinen versuchen anspruchsvollere Konzepte zu entwickeln, wenn sie sich erwa im Wirtschaftsrecht bei marktbeherrschenden Unternehmen über $\$ 22$ GWB auf die Suche nach swettbewerbsanalogen Preisen machen ${ }^{6 / 1}$ oder im Steuerrecht , Als-ob-Wenbewerbs - Preise an die Stelle von konzerninternen Verrechnungspreisen setzen wollen ${ }^{6}$. Weit davon entfernt, sich dem Problem der Wertschöpfung zuwenden zu können, peilt die Dogmatik des Bereicherungsrechrs bisher nicht einmal diese raufgeklärtec Ebene der Preisbildungsdiskussion an, sondern beharn aul dem Streit um rex rer oder ex negoriatione, allerdings mit dem Gewinn, die Unzulänglichkeiten der,$A l s-o b-$ Wertbewerbs-Annahmen nicht angehen zu müssen.

\section{Bereicherungsreche als Verfahren}

Wo derartig beharrlich, verbissen, auch mal ironisch gestritten wird, wird geraten, sich bescheiden zu geben und einzuräumen, daß man, $d$. $h$. der wissenschafuliche Erkenntrusstand insgesamt, noch nicht so weit sei, um wirklich theoretisch fundierre Ergebnisse zu liefern. Es sei nörig, ökonomisch-theoretische Erkennenisse fiuchtbar zu machen, was dadurch erschwert werde, daß auch die Okonomie-Theorie noch nicht so weit se ${ }^{-66}$. Inzwischen - Entscheidungszwang des Juristen - gelte es, pragmatisch zu verfahren.

Bei aller Sympathie für Bescheidenheit kommt diese Aussage überraschend, besagt sie doch, daß die großen Theoretiker des Zivilrechts - und sie waren es, die den Kampf ums Bereicherungsrecht geführt haben - bisher unzulänglich gedacht haben trotz aller ausdrücklichen Versuche, ökonomische Theorie in ihre Interpretarionen mit aufzunehmen. Bevor wir uns auch diesem Verdikt anschließen, wollen wir die These prüfen, daß die Vergeblichkeit, im Bereichenungsrecht za einem Wertungskonsens zu gelangen, nicht der theoretischen Unzulänglichkeit der Forscher anzulasten ist, sondern sich als reale Vergeblichkeit entpuppt angesichts einer gesellschaftlichen Realität, die sich dagegen spern, materiale Gerechuigkeitskriterien oder auch nur Funkrionalitätskriterien in den Beziehungen der .Privarrechtssubjektec zueinander ex-ante, planvoll zu bestimmen und die diese Sperre auch nicht vor den Zauberformeln von 'Macerialisierung، und 'public policy ${ }^{67}$ zurückzieht. Das gilt selbstverständlich für die Berücksichrigung der Produktion von Werten, die für die Legitimation der Wert-Zuordnung keine Rolle spielc. Obwohl sich etwa bei Schulz die apokryphe Aussage finder, "daß weder der Preis noch der Wert in der Sache drinstecken und daher auch nicht aus der Sache fließen können ", sondern „durch die schärzende menschliche Tätigkeit « geschaffen werden ${ }^{68}$, kann die produkrive 'menschliche Tärigkeitı nicht zur Auslegung von Gesetzen in der Marktökonomie beitragen. Souverän herrscht die tote Arbeit über die lebendige. Wo ,Arbeit‘ zum

64 BGHZ 68, 25; ausführlich Knieper, Weltmarkt, Wirtschaftsrccht und Nationalstaxt, 1976, S. 246 ff.

6s Auslührlich Knieper (FN 64) S. I 49 if.

66 Joerges (FN 1) S. 7 f./65; Esser-Weyers (FN 8) 5. 321.

6) Jocrges (FN 1) S. 66/68.

68 Schulz (FN 6 ) S. 


\section{Kurskorrekturen}

\section{im Recht}

Die Vorträge und Referate

des Deutschen Richtertages 1979 in Essen

Herausgegeben vom Deutschen Richterbund

1980. VIII, 327 Seiten, kartoniert DM 89,-

ISBN 3-452-18733-0

Die Dokumentacion der Vorträge und Referate, die auf dem Deutschen Richterlag 1979 in Essen gehalten worden sind, entspricht dem vieliach geäußerten Wunsch, dic Gedanken und Anrcgungen der Veranstaltung in gesammeler Form der justizpolitisch interessierten Offentlichkeit zugänglich zu machen.

Das nachhaltige Echo, das der Richtertag in Fachkreisen gefunden har, zeigr sich insbesondere in der Tatsache, daß die Diskussion der in Essen angeschnittenen Fragen aus Stcuerrecht, Strafrecht und Verfahrensrecht inzwischen sowohl im Verband selbst als auch im parlamentarischen Bereich und in der Ministerialbürokratie mit Intensität lorrgesetzt wird. So ist beispielswcise zu erwarten, daß der Gesetzgeber in abschbarer Zeir konkrete Vorschläge zur Reform des Ordnungswidrigkeitenrechts vorlegen wird. Auch in der akruellen Auseinandersetzung um den Fortbestand bzw. die Rücknahme einzelner Vorschriften des Straf- und Strafprozeßrechts finden die auf dem Richtertag vorgebrachten Argumente Beachiung. Für den innerverbandlidhen Bereich schließlich hat der Richterrag wesentliche Oriencierungspunkte für die Erarbeitung justizpolicischer Leitlinien des Deutschen Richrerbundes gesctzt.

Insgesamt zeigt sich, daß die Themen des Deurschen Richtertages 1979 von erheblicher justizpolicischer Tragweite sind. Die Dokumentation wird ihren Zweck erfüllon, wenn sie hilft, bei der notwendigen weitergehenden Erörterung grundsätzliche Fragestellungen nichr aus den Augen zu verlieren.

\section{Carl Heymanns Verlag Köln Berlin Bonn München}


Problem wird und dem ,Kapiral gegenübergestellt wird ${ }^{69}$, ist die ,Arbeit des Händlers, seine Verkaufsgeschicklichkeit am Markı gemeint.

Diese scrukturelle Trennung von Produktion und Verwercung wird in der juristischen Diskussion nicht nur verteidigt, sondern so weit wie möglich verstärkt. Die Verteidigung durchdringt die bereirs zitierte Aussage v. Caemmerers, nach der die Leistung des abhängig Beschäfrigten die Leistung des Unternehmers ist. Sie setzt sich fort in der einhelligen Incerpretarion des über \95 r BGB Bereicherungsansprüche begründenden $\$ 950$, die ebenfalls dekretiert, daß der „Geschäftsinhaber « die "zur Herstellung der neuen Sachen erforderliche Arbeit « leistet ${ }^{\circ}$. Im gewerblichen Rechtsschurz gilr als „Urheber der MSusrer und Modelle« im Sinne des Geschmacksmusrergeserzes nicht der Urheber, sondern der ${ }^{2}$ Eigentümer* $(\$ 2)$. Die Erfindungen, die in der Bundesrepublik von abhängig Beschäfrigten gemacht werden - das sind $90 \%$ aller Erfindungen ${ }^{71}$ - tönnen vom "Arbeitgeber 4 in Anspruch genommen werden ( $\int S 6 / 7$ ArbNEriG), wobei noch der größere Teil der in der Freizeit gernachten Erfindungen diesem Zugriff unterliegt, da der Bereich der ,freien Erfindungenc sehr eng ist $(\$ \$ 18 / 19 \text { ArbNErfG })^{72}$.

Nachdem als eherner Grundsatz festliegt, daß die Wersschöpfung als Wertungskriterium für die ,Verteilung neu produzienter Wertec keine Rolle spielt, nachdem die Brechrsche Frage des idenkenden Arbeiters, wer das siebentorige Theben gebaut habe, eindeutig zugunsten des Königs beantwortet ist, soll die juriscische Doktrin das Kunststück vollbringen, Königreiche, Domänen, Vermögenspositionen nach Kriterien materialer Gerechtigkeit zuzuteilen; indem sie gängige Interprecationsmethoden wie den Rückgriff auf die ,Positivitäı ı des Gesetzeswortlauts gering achtet, gibr sie vor, dazu in der Lage zu sein. Damit muß sie das Verhalten der sich in Konkurrenz aufeinander beziehenden Wareneigentümer mir einer ex-ante-Bewerrung qualifzieren, obwohl die ,Richrigkeice der Konkurrenz sich nur vom Resultat aus messen läßr. Konkreter: In der Auseinanderserzung um die Bereicherungsansprüche im Dreiecks-Verhältrnis war offensichtlich geworden, daß der Versuch einer individuellen Zuschreibung des (Konkurs-)Risikos - gleichgültig ob über offene Wertung oder über die Subsumtion unter den Leistungsbegriff - keinen Maßstab für Richtigkeit an der Hand hat, da ein gesellschaftliches, ökonomisches, konjunkturelles Risiko auch über eine Funktionsbestirnmung »der einzelnen Leiscungsbeiträge" nicht individualisiert werden kann"s. Auch die Hoffnung auf zukünftige "Anleitung durch sozialwissenschaftlich-ökonomische Handlungskonzepte $\alpha^{74}$ hilft da nicht weiter. Im gerverblichen Rechusschutz, dem zentralen Problembereich der ${ }^{\text {Ein- }}$ griffskondikcions, war die den Bereicherungsanspruch Kohlers ablehnende Begründung zitiert worden, daß Immaterialgüterrechte der Allgemeinheit zustreben', wobei zu ergänzen ist, daß sie auch aus ider Allgemeinheit< entstehen. Wie beim Dreiecks-Verhältnis schimmert in dieser Bemerkung die Gesellschaftlichkeir der Reichrumsgewinnung auf, die im kapitalistischen Privateigencum und der Konkurrenz sogleich wieder verschwindec. Das Scheicern von Versuchen aber, kapitalistisches Privateigentum material zu legirimieren - über eigene Arbeic, über Vernunfr, über Leistung - habe ich an anderer Stelle ausführlich begründet: es ist staadich geschürzes gesellschaftliches Gewaltverhältnis"s. Entsprechend vergeblich isc es, die

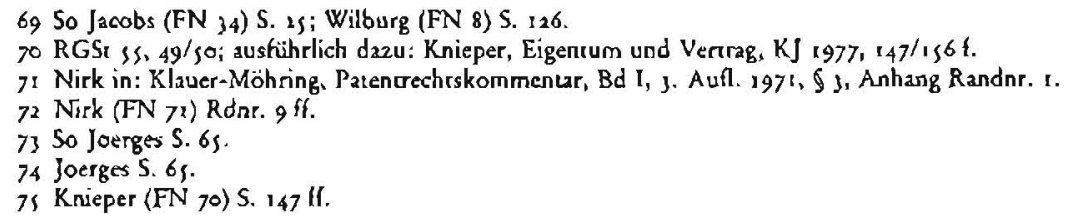


Konkurrenz der Privateigentümer, in der sich Verwertung vollziehr, durch Kriterien materialer Gerechrigkeit zu legicimieren und zu kanalisieren sowie die Norwendigkeit der Verwertung, des W/arenumschlags mit der Nortvendigkeic des Habens zu harmonisieren.

Die Versuche aber, Bereicherungsansprüche theorecisch zu fundieren, müssen eben das strukcurell Notwendige immer wieder als das individuell Verantworteze ausgeben. Sie unterstellen, daß der Eigentümer einer Ware substantiell frei sei, sie zu veräußern oder zu behalcen; die Definition des Eigenturns scellt den Ge- und Verbrauch, das suti, fruic gleichberechtigt neben die Veräußerung - als könnte der Bauscofflieferant, anstatt die Ziegel zu veräußern, sie selbst benurzen, als könnte die Bank, anstatc Geld zu verieihen, es einem anderen nüzzlichen Zweck zutühren, als könnce die Fluggesellschaft den vom Minderjährigen eingenommenen Platz selbse einnehmen. In Wahrheit kommt das Eigencum erst „durch den Vertrag zustande ${ }^{76}$, der Zuordnungsgehalt des Eigentums wie jedes anderen dinglichen Rechts realisier sich erst in der Geschäftsbeziehung. Das Ziel der Geschäfrsbeziehung aber ist nicht die Durchsetzung des sf reien Willens - der emphatische Ausdruck der $¥$ Selbstbestimmung a zur Kennzeichnung des Zivilrechts ${ }^{7}$ übertönt nur leicht den Zwang zur "Selbstdisziplinierung ${ }^{78}$-, sondem die nuczbringende Verwendung von Eigentum. Doch manifestiert sich im vertragsförmlichen Hin und Her nicht nur kein freier Wille, sondern ebensowenig die Durchserzung einer inhaltlich bestimmren gesellschaftlichen Ordnung. Um so weniger lassen sich aus den strukrurellen Bedingungen richtigkeitsgewährende Kriterien zur Lösung juriscischer Einzelfälle ableiten.

In der widersprüchlichen deus-ex-machina-Rolle, die der ffreie Willernoch in seiner verkümmerten juristischen Ausprägung spiełt, komme diese Beziehungslosigkeit zum Ausdruck. Das gilt für die Vertragskategorie selbst: Das geschlossene Konzept eines Zivilreches als fast reiner Durchseczung des Willensprinzips bei v. Savigny hat praktisch nie Bestand gehabt und ist auch theoretisch vollstindig durchlöchert. Von den Vertrerungsregeln ${ }^{7^{82}}$ über die Nichranerkennung des geheimen Willensvorbehalces (\$ I1 6 BGB) zur Nichtberücksichrigung des Motivirrtums bei der Anfechrung ( $\$$ i g B B B) kennt bereits das BGB selbst ,Durchbrechungen des Willensprinzips, und es hac wenig Mühe bereitet, diese Durchbrechungen von der Anerkennung eines allgemeinen Vertrauensprinzips bis hin zum faktischen Vertag zu erweitern. In der offen instrumentellen Verwendung in $\$ 2$ AGB-Gesecz, in $₫ 22$ GWB ist denn auch das Axiom der Willensfreiheit geserzlich ad acta gelegt. Im Bereicherungsrecht setzr sich der instrumentelle Einsacz des sfreien Willens, fort:

- So ist einerseits akzeptiert, daß die subjektive Aquivalenz<, die im vertraglich vereinbarten ,Ware gegen Geld, niedergelegt sei, jeden Bereicherungsanspruch ausschließr ${ }^{79}$, andererseits wird auch an dieser Äquivalenz festgehalten, wo zum mindesten ein Vertragspartner einem zur Anfechtung berechtigenden Irrum unterlag: Wenn wechselseitig Leistungen zurückzuerstatren sind, wird ein saldo gebildet oder - nach einer neueren Auffassung - ein , fakcisches Synallagmar konstruiert ${ }^{80}$, das über die Gükcigkeit der Willenserklärungen hinaus Bestand hat.

- So wird bei Fehlen einer vertraglichen Abrede eine nangemessenea Entschädigung

76 Hegel, Grundlinien der Philosophie des Reches, 1821, $\$ 72$.

7) Mesmicker, Macht - Recht - Wircschafesverfassung (1973), ziviert nach Mestmäcker (FN 21) S. $\because \mathrm{ff}, 1 \mathrm{x}$.

78 Elias, Uber den Prozeß der Zivilisacion, 2. Aufl. 1977, Band II, S. 339, 2uch: S. J19 ff.

$78 \mathrm{a}$ In jungerer Zeit hat besonders Müller-Freieniels, Die Veriretung beim Rechisgeschäle, 1955, bes, S zo9 ff. auf den Widerspruch von Privalauconomie und Verierung aufmerksam gemache.

79 Dazu erwa Rothoch, Vermögensverlust und Bereichenungsausgleich, AcP 163 (1963), 215/221; Beu. thien (FN 50) \$818 Anm. \& d.

8o Dazu eiwa Esser-Weyers (FN 8) S. 98 f.; v. Cacmmerer (FN 8) S. 260 ff. 
danach berechnet, wie sie ein durchschnirtlicher Vertragsparner, $d$. h. Realisierer des freien Willens geltend machen würde.

- So wird das Problem der saufgedrängten Bereicherunge vom "Standpunkr des objektiven Berrachters aus ${ }^{81}$ gelöst, der gerade nicht einen Willen, sondern wirtschaftliches Kalkül verkörpern soll.

- So nimmt die heute h. M. bei der Beurreilung von Wucherdarlehen und -mieren im Rahmen des $\$ 8 \mathrm{r}, \mathrm{BGB}$ an, daß die dem ,freien Willen, entsprechende WucherVergütung auf ein angemessenes $\mathrm{Ma} B$ zu reduzieren $\mathrm{se}^{; 82}$.

- So gehört zu den wesenclichen Gründen der dogmatischen Trennung von ,Leistungs - und 'Eingriffsi-Kondiktion, die wsuggestive Macht der Leiszungskondikcionen $\alpha$ zu durchbrechen, die durch das Moment des Leistungswillens gekennzeichnet seien $^{8}$, um dann in der Zuordnungs- wie der Rechrswidrigkeitslehre inmer wieder auf ,quasi-contractlicher Annahmen zurückzufallen, sei es, um ordnungsgemäßes Verhalten als Pflicht zum Vertragsabschluß oder die Rechtswidrigkeit als vertragsloses Handeln zu charakterisieren. Als - beliebiges - Beispiel sei wieder die Arbeir von Kleinheyer herausgegriffen, der gegen ,quasi-contractlichesc Denken polemisiert, um es dann doch über die Konstruktion des venire contra facrum proprium und die Annahme, daß̉ ein "Verkehrswert ... als Preis auch vereinbart wäre*, wieder einzubeziehen ${ }^{84}$. Diese Beispiele belegen wiederum weniger theoretische Inkonsistenz als die Tarsache, daß bei aller Uberwindung von Saivgny auf den Willen als Legitimationskrücke immer wieder zurückgegriffen wird, wobei gerade der Legitimacionszweck die instrumentelle, beliebige Verwendung erklärt. Ebenso wie sich vom quasikontraktlichen Charakter des Bereicherungsrechts sprechen läßt, Ließe sich vom Quasikondikrionen-Charakter des Verragsrechrs sprechen. In beiden Gebieten geht es nicht um Willens-, sondern um Verwerrungszusammenhänge mit der Foige, daß Vertrags- und Bereicherungsansprüche tatsächlich weitgehend austauschbar sind, wie sich am Vergleich von BGHZ 21,319 (Parkplatz-Fall über fakcischen Vertrag) und BGHZ 20, 270 (Parkplatz-Fall über $\$ 8 \mathrm{r}_{2}$ ) zeigt. Die positive Kritik ${ }^{85}$, die der Flugreise- Fall provoziert hat, weil in ihm - in Pecer-Stuyvesant-Manier - der Duft der weicen W/elt einer Flugreise die Sozialtypik der schnöden U-Bahnfahr abgesprochen worden isr, reduziert sich insoweit praktisch zur Marginalie.

Allgemeiner: In der Konstrukrion des ,fakrischen Synallagmas bei der Leisnungskondikcion wird die Orientierung des Bereicheningsrechts am Verrag ebenso deutlich wie bei der fikriven Pflicht zum Vertragsschluß als Qualifikation des ordnungsgemäßen Vorgehens und als Basis zur Berechnung der Anspruchshöhe bei der Bereicherung in sonstiger Weise،. Die Nähe beider Anspruchsrypen zum Vertragsrecht aber läßt die feinen Ziselierungen, mit denen sie gegeneinander ausgespielı werden, tatsächlich als inhaldlich überflüssig erscheinen. Gewicht erfähr die Differenzierung ja ohnehin erst mit der Annahme, daß der freie (Leistungs-) Wille ein respektables Interpretationselement sei. Reduziert man diese Ansicht erst einmal aut ihren instrumentellen Gehalt, verliert das System der Anspruchsuncerteilungen sein Gewicht.

Wo materielle Legitimarionskriterien fehlen, um das positive Recht richug auszulegen, können genausogut soziale Kriterien herangezogen werden: Nehmen wir den Flugreise-Fall wriedenum als Beispiel: Keine sBindung ans Gesetz، hätte den BGH

81 Koppensteiner-Kramer (FN 1) S. 174.

8. Medicus, Bürgerliches Recht, 6. Aufl. 1973, 5. 304.

83 Wilburg (FN 8) S. is

84 Kleinheyer (FN 60) S. 473.

85 Canaris (FN 63): vgl. auch Mestmäcker (FN 21) S. 451 ff. 
gehindert, den minderjährigen Schüler, der von seinen auch durch Flugreklame geschürten Träumen verleicet nach New York flog, vom Bereicherungsanspruch der Lufufahrtgeselischaft zu entlasten - im Gegenteil, es war mühevoll genug für den BGH, trotz des ,Luxus-Charakrers der Reise und gegen die Tatsache, daß die Maschine nicht ausgebucht war, einen Anspruch zu begnünden. In der Anwendung der deliktsrechrlichen Einsichrsfähigkeits-Bescimmung (\$828) trotz der Charakterisierung als Leistungsverhältnis und in der beinahe hämischen Bemerkung, es sei davon auszugehen, „daß die Mutter des Beklagten das nach den gegebenen Umständen jeweils Beste für ihren Sohn im Auge hatte" ${ }^{86}$, kommt eine penetrante, kleinbürgerliche Mischung von Poena und Pädagogik, von erhobenem Zeigefinger gegen Mutter und Kind zurn Ausdruck. Es wäre sicherlich weder rationaler noch irrationaler, mehr oder weniger durch den Gesetzeswortiaut geboten gewesen, der ohnehin höchstens um einen Plastik-Snack »entreicherten* Fluggesellschaft den Bereicherungsanspruch abzuerkennen - mit Sicherheit aber wäre es gütiger gewesen.

Wer die Analyse des Zivilrechts aul seinen materiellen Teil beschränkt, d. h. die verfahrensrechrliche Dimension außer acht läßt, wird die hier vorgestelle Auffassung von der Vergeblichkeit der Suche nach richrigem Bereicherungsrecht ais Beleg für die Itrarionalitäı und Sinnlosigkeit der Diskussion werten. Dieser Schluß ist jedoch voreilig, wenn ihm auch durch die traditionelle Trennung von materiellem und Verfahrensreche Vorschub geleister wird: Die kühnen dogmatischen Konstruktionen des materiellen Rechts gedeihen gerade in der Abkapselung. Beziehen wir die Verfahrensziele des Zivilprozesses in die Debatte ein, dann werden Sinn und Racionalität der Bereicherungsrechtsdiskussion deutlich, wobei allerdings der Aspekc des Diskussionsverfahrens sich vor seinen Inhalt schiebt. Zur Erläurerung dieses Gedankens nehme ich meinen Ausgang bei Luhmann, der in einer ausführlichen Analyse die Einsicht vorgetragen hat, daß die „Funktion des Verfahrens... die Spezifizierung der Unzufriedenheit und die Zersplitcerung und Absorption von Protesten ${ }^{87}$ sei. Dem Verfahrensbeteiligten sei die Möglichkeit genommen, nseine Interessen als konsensfähig zu generalisieren und größere soziale und politische Allianzen für seine Ziele zu bilden ${ }^{89}$. Er werde isoliert, der Konflikt individualisiert; wesentliches Ziel des Verfahrenszeremoniells sei es, den Verlierer, der sich gegen das Ergebnis auflehnt, zum Sonderling und Querulanten abstempeln zu können ${ }^{89}$.

Der Zusammenhang zwischen Bereicherungsrechesdiskussion und Verfahuen wird deutlich: Ich harte entwickelt, daß die Individualisierung von Vermögenszuteilungen auf diese oder jene Partei substantiell nicht gerechtfertigt werden kann. Die anhaltende, mir Ernst und Seriosität geführte Debarte stellt nun gerade jenes Zeremoniell dar, daß wenn schon nicht substantiell korrekce Kriterien für individuelle Zurechnung anzugeben vermag, dennoch gerade in der Aufrechterhalung von Unsicherheit die Szene für faktische Individualisierung, d. h. Isolierung im Prozeß vorbereitet. Im Bereicherungsrecht besteht um so mehr Anlaß für elaborierte, süberzüchtete", "verwirrende Konstrukrionen, als dic einfacheren Verantwortungszuschiebungen auf den selbstverantwortlich, Handelnden, auf die im Vertragsrecht gebaut wird, im Bereicherungsrecht nicht zur Verfügung stehen. Erst im Verfahren wird die Individualisierung komplert, um die das materielle Rechr sich vergeblich bemüht.

$86 \mathrm{BGH} \mathrm{JZ} 197 \mathrm{r}, 556 / 560$.

87 Lubmana (FN 4) S. 116.

88 Luhmano (FN 4) S. 117.

89 Luhmann (FN 4) S. $117 \mathrm{ff} / 33$ t. 


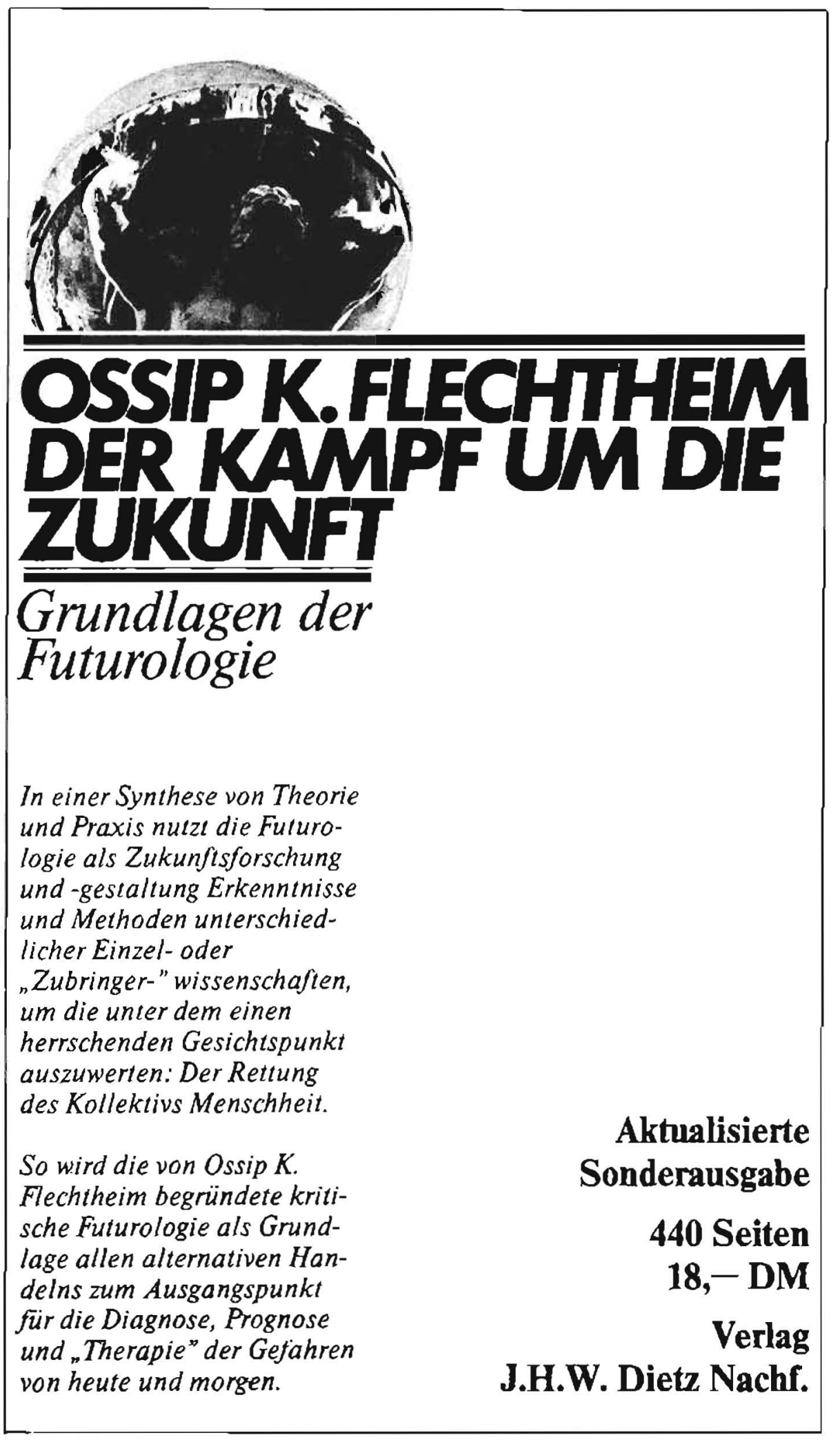

